\title{
Fast Screening of Olfactory Function Using the Q-Sticks Test
}

\author{
Agnieszka Sorokowska a, b Anna Oleszkiewicz ${ }^{a, b} \quad$ Amir Minovi $^{c}$ \\ Claus Günther Konnerth ${ }^{d}$ Thomas Hummel ${ }^{a}$ \\ asmell \& Taste Clinic, Department of Otorhinolaryngology, TU Dresden, Dresden,

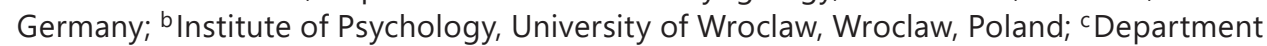 \\ of Otorhinolaryngology, Head and Neck Surgery, Ruhr University Bochum, St. Elisabeth \\ Hospital, Bochum, Germany; ${ }^{d}$ Karl-Hansen Medical Center, Department of Otolaryngology, \\ Head and Neck Surgery, Bad Lippspringe, Germany
}

\section{Keywords}

Olfaction · Olfactory disorders · Testing · Anosmia · Normosmia · Q-Sticks test

\begin{abstract}
Objectives: Classical, longer olfactory tests are associated with difficulties in everyday practice. The aim of this study was to investigate the clinical utility of the Q-Sticks test within the context of a multicenter study. Methods: A total of 333 subjects, aged between 12 and 88 years, completed a 3-item Q-Sticks test and a longer Sniffin' Sticks identification test. We analyzed the sensitivity and specificity of the test results for different cutoffs for anosmia and hyposmia. Results: Our study showed that the test can be used on subjects aged 12 years and older, regardless of their sex. The data indicated a test sensitivity and specificity of 91.8 and $92 \%$, respectively, with a score of 2 taken as a cutoff for olfactory dysfunction. Conversely, normal olfactory function can be declared with high probability with a score of 3 in the QSticks test. Conclusion: We reconfirmed that the Q-Sticks test is a good screening tool for everyday medical practice and in laboratory settings, although it has to be kept in mind that the test can produce false-negative results at a score of 3 . The test was relatively sensitive to anosmia and differences between normosmic and hyposmic/anosmic patients.
\end{abstract}

(c) 2019 S. Karger AG, Basel 
Sorokowska et al.: Olfactory Screening with the Q-Sticks Test

\section{Introduction}

Olfactory deficits are frequent in the general population [1], which is particularly important given the relationship between olfactory loss and many types of neurodegenerative diseases, including idiopathic Parkinson's disease [2]. Olfactory problems are also often associated with depression [3]. However, olfaction is not only a marker of different disorders, it also plays an important role in everyday life. For example, the sense of smell contributes to healthy lifestyle, since aromas are one of the aspects of rewarding qualities of food [4]. Further, odors are known to have influence on the perception of pain [5] and were shown to affect mood [6]. All these aspects and correlates of olfactory performance hint the overall importance of olfactory testing.

There are numerous tools to assess olfactory function [7], with the Sniffin' Sticks Test (SST) $[8,9]$ being one of the most popular among them. This test comprises a battery of three different tools: olfactory threshold, discrimination abilities, and identification test. The SST enables a detailed diagnosis of olfactory dysfunctions, especially when the extended versions of some subtests are applied [10]. However, detailed testing of all patients' olfactory abilities in nonspecialized medical centers or during an introductory ENT diagnosis is not always possible. Given the prevalence of olfactory loss in the general population [11], it seems a particularly important problem in everyday clinical practice, but also in clinical research, where the sense of smell does not have to be a central point of interest, but anosmia or hyposmia are frequent exclusion criteria for the subjects.

The identification test procedure is the simplest and shortest of the three SST subtests [8]. Identification tests are easily understood by both the patient and the experimenter [12]. In addition, identification scores can be related to the cognitive performance of subjects [13], which makes them a valuable tool for research in non-ENT specializations. As low olfactory identification often suffices to observe olfactory dysfunctions [14], for screening purposes, a relatively wide array of olfactory identification tests is in use. Some of these tools are based upon the SST identification test. First of all, one might simply use the 16-item odor identification test [8]. Application of this subtest as an abbreviation of the full SST seems a reasonable solution in laboratory or clinical settings, since this test takes about 6-10 min. However, considering the average time a doctor can spend with a patient, this appears to be still lengthy for clinicians, and thus there are also other, shorter versions of this test. The most popular, shortened version of the SST identification test comprises 12 odorants [14]. There are also 8-item [15] and 5-item identification tests [16], but the time pressure and other important limitations of everyday clinical practice led to the creation of an even shorter screening tool, the so-called Q-Sticks test [17].

In the Q-Sticks test, three odors (cloves, coffee, and rose) are presented in felt-tip pens. These odors were selected because they are widely known and their identification is not strongly dependent upon subjects' age [17]. Thanks to the simplicity and short duration of the procedure, the Q-Sticks test seems to be a good solution in everyday medical practice and in laboratory settings. Importantly, more sophisticated olfactory testing across Europe is often performed with the use of the extended version of the SST, which allows for a more detailed diagnosis of olfactory dysfunction. The present study aimed to investigate the clinical utility of the Q-Sticks test within the context of a multicenter study in three German university hospitals.

\section{Subjects and Methods}

Participants

The study participants were 333 people whose age ranged from 12 to 88 years ( mean $=49.5$, SD $=16.1$ ). Of those, 167 were women aged between 12 and 88 years (mean $=49.9, \mathrm{SD}=15.8$ ) and 166 were men aged 
Sorokowska et al.: Olfactory Screening with the Q-Sticks Test

between 15 and 83 years (mean $=49.1$, SD = 16.4). The study was conducted in three ENT clinics across Germany. The participants were either recruited among the patients of these clinics or enrolled as healthy volunteers to take part in the project as a control group.

Testing Procedure

The subjects were presented three odorant-filled felt pens which were briefly opened to release the smell (for more details on the SST see Hummel et al. [9]). By means of a multiple-choice task, identification of odors was performed from a list of four descriptors each. Because of the problems associated with selfratings of olfactory function, participants' olfactory function was additionally assessed birhinally with the SST odor identification test $[8,9,15]$. Participants completed the 12- or 16-item tests.

\section{Statistical Analyses}

In the data analysis, we used two test scores for each subject: (a) the result in the Q-Sticks test (i.e., score 0 [no odor identified] to 3 [all odors identified]) and (b) the 16-item SST result (score 0-16) or the 12-item SST result (score 0-12). Statistical analyses were performed by means of SPSS vs. 21 (SPSS Inc., Chicago, IL, USA) with the alpha level set at 0.05 .

We performed independent-sample $t$ tests to investigate sex differences in odor identification across all measures. Further, we computed $\chi^{2}$ tests to compare the numbers of functionally anosmic and normosmic subjects with Q-Sticks outcomes of $0,1,2$, or 3 correct odor identifications. Then, Q-Sticks outcomes of 0,1 , 2 , or 3 correct odor identifications were compared with the scores in the 12- and 16-item tests by means of univariate analyses of variances, with Q-Sticks result as a between-subject factor (4 levels) and age of participants treated as a covariant. We also compared the Q-Sticks result with the proportion of correct answers in both identification tests (12 and 16 items) across age groups.

Finally, we analyzed the sensitivity and specificity of test results for different cutoffs using the following formulas: sensitivity $(\%)=100 \times$ correctly positive $/($ correctly positive + false negative $)$; specificity $(\%)=$ $100 \times$ correctly negative / (correctly negative + false positive) [17]. This was done (a) for anosmia and (b) for the diagnosis of normal or pathologic (i.e., anosmia and hyposmia) olfactory function.

\section{Results}

A total of 196 participants completed the full SST identification test and 137 completed the 12 -item version. The results in the 16-item identification test ranged from 0 to 15 (mean $=7.3, \mathrm{SD}=3.9$ ), and in the 12 -item test the scores ranged from 0 to 12 (mean $=9.9$, $\mathrm{SD}=2$ ). The results in the $\mathrm{Q}-$ Sticks test ranged from 0 to 3 (mean $=1.9, \mathrm{SD}=1.1$ ).

\section{Sex Differences}

The results of the independent-sample $t$ tests revealed no significant differences between scores obtained by men and women in the 16-item identification test, the 12-item identification test, or the Q-Sticks test ( $p$ values > 0.05). Therefore, the analyses were performed for men and women together.

\section{Olfactory Testing}

We examined whether subjects who obtained lower scores in the Q-Sticks test had also lower olfactory performance in (a) the full SST identification test and (b) the 12-item SST identification test. According to the results of the two longer identification tests, $40.2 \%$ of the participants were functionally anosmic (score $<9$ in the 16 -item identification test or $<7$ in the 12-item identification test), $22.5 \%$ were hyposmic (score $9-12$ in the 16 -item identification test or 7-9 in the 12-item identification test), and 37.2\% were normosmic (score $>12$ in the 16 -item identification test or $>9$ in the 12 -item identification test). 
Sorokowska et al.: Olfactory Screening with the Q-Sticks Test

Table 1. Numbers of functionally anosmic, hyposmic, and normosmic subjects (according to their identification scores in the 12- and 16-item identification tests) scoring $0,1,2$, or 3 in the Q-Sticks test

\begin{tabular}{llccr}
\hline $\begin{array}{l}\text { Q-Sticks } \\
\text { result }\end{array}$ & $\begin{array}{l}\text { Functional } \\
\text { anosmia }\end{array}$ & Hyposmia & Normosmia & Total \\
\hline 0 & 64 & 1 & 0 & 65 \\
1 & 33 & 7 & 5 & 45 \\
2 & 26 & 35 & 25 & 86 \\
3 & 11 & 32 & 94 & 137 \\
\hline Total & 134 & 75 & 124 & 333 \\
\hline
\end{tabular}

We observed a tendency to identify fewer odors in the Q-Sticks test among subjects with low comprehensive SST identification test results (Table 1). Subjects with low scores in the comprehensive SST identified fewer odors in the Q-Sticks test.

Functional anosmics more frequently obtained lower scores in the Q-Sticks test and normosmics more frequently obtained higher scores $\left(\chi^{2}\right.$ test: $\left.p<0.001\right)$. This remained true for the comparison of anosmic, hyposmic, and normosmic participants $\left(\chi^{2}\right.$ test: $\left.p<0.001\right)$. The same was observed when comparing the Q-Sticks results of subjects with pathologic olfactory function (anosmia or hyposmia) and a normal sense of smell ( $\chi^{2}$ test: $\left.p<0.001\right)$. The same results were obtained when men and women were analyzed separately $\left(\chi^{2}\right.$ test: $p<$ 0.001 for both groups).

We found a significant main effect of Q-Sticks result $\left[F_{(3,132)}=41.9, p<0.001\right]$ and of age $\left[F_{(1,132)}=19.8, p<0.001\right.$, adjusted $\left.R^{2}=0.57\right]$ on the 12 -item identification test score. The same was true for the 16-item identification test $\left[F_{(3,191)}=83.4, p<0.001\right]$, with a significant effect of age $\left[F_{(1,191)}=6.1, p<0.05\right.$, adjusted $\left.R^{2}=0.56\right]$, and for the proportion of correct answers in either the 12- or the 16-item identification test $\left[F_{(3,328)}=162.5, p<0.001\right.$, age $F_{(1,328)}=$ 56.1, $p<0.001$, adjusted $\left.R^{2}=0.65\right]$.

Across all age groups, the Q-Sticks result was a significant predictor of proportion of correct answers in the identification test [for group aged 12-35 years: $F_{(3,66)}=57.4, p<0.001$, adjusted $R^{2}=0.71$; for group aged $36-55$ years: $F_{(3,132)}=93.2, p<0.001$, adjusted $R^{2}=0.67$; for group ages $>55$ years: $F_{(3,123)}=41.8, p<0.001$, adjusted $R^{2}=0.49$ ].

\section{Diagnosis of Anosmia and Hyposmia}

Functional anosmia was identified as 0 correct Q-Sticks identification at a test sensitivity of $47.8 \%$ and a specificity of $73.9 \%$ (Cohen's kappa $=0.21$ indicating fair agreement between all involved tests). This result remained unchanged when analyzing men and women separately (Cohen's kappa $=0.22$ and 0.20 , respectively) and across age groups of 12-35 years, $36-55$ years, and $>55$ years (Cohen's kappa $=0.22,0.22$, and 0.21 , respectively).

When we used a score of 0 or 1 to discriminate anosmia from hyposmia and normosmia, the test sensitivity was $72.4 \%$ and test specificity equaled $83.4 \%$ (Cohen's kappa $=0.29$ indicating fair agreement). In this case, the results for men and women were similar (Cohen's kappa = 0.25 and 0.33 , respectively), and this was also true for the analyzed age groups (12-35 years: Cohen's kappa $=0.18 ; 36-55$ years: Cohen's kappa $=0.30 ;>55$ years: Cohen's kappa $=0.33$ ).

The test sensitivity and specificity equaled 91.8 and 92\%, respectively, when a score of 2 was treated as a cutoff for the diagnosis of anosmia versus hyposmia and normosmia (Cohen's kappa $=0.20$ indicating fair agreement).

When hyposmia was identified by two correct Q-Sticks identifications, sensitivity was $46.7 \%$ and specificity was $40.7 \%$ (Cohen's kappa $=0.11$ ), by two or three identifications it was 89.3 and $30 \%$, respectively (Cohen's kappa $=0.29$ ), and by three identifications the numbers were 42.7 and $23.4 \%$. 


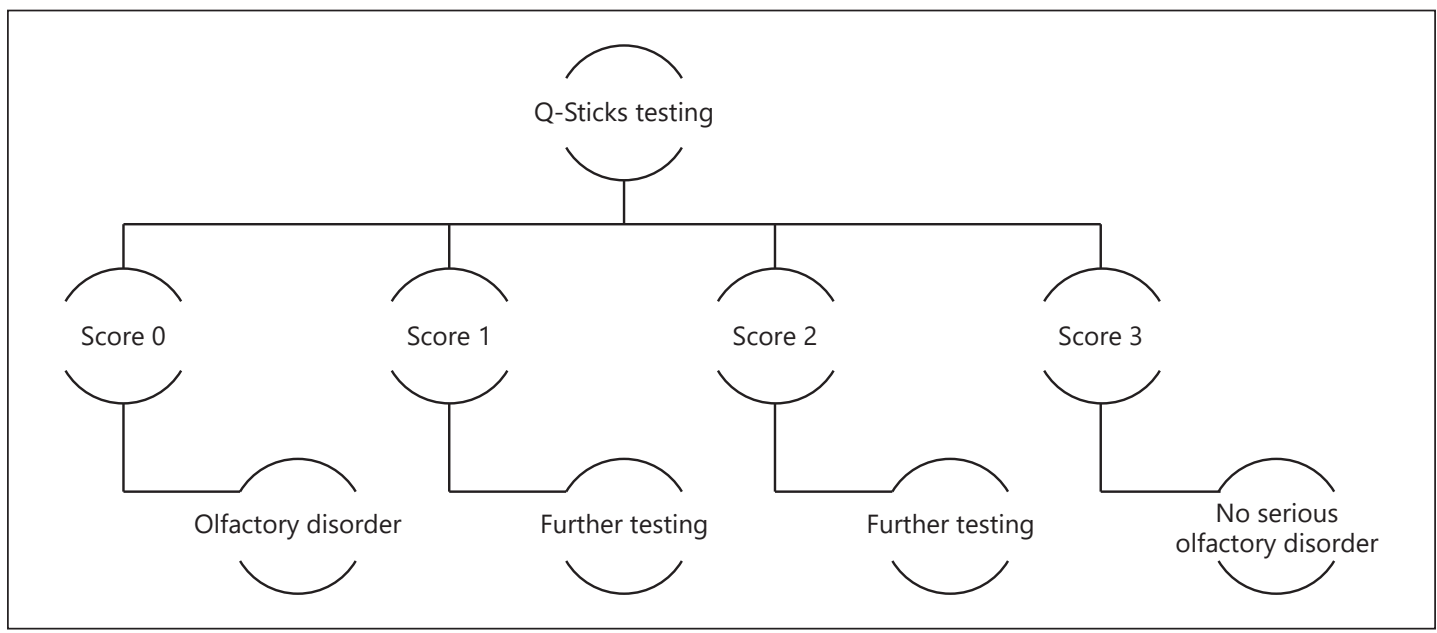

Fig. 1. Proposed diagnostics based on Q-Sticks scores.

\section{Diagnosis of Normal Olfactory Function}

Conversely, normosmia was identified by three correct Q-Sticks identifications at a test sensitivity of $79.4 \%$ and a specificity of $68.6 \%$ (Cohen's kappa $=0.20$ ). When normosmia was identified by two or three correct Q-Sticks identifications, sensitivity was $50.2 \%$ and specificity was 53.4\% (Cohen's kappa $=0.29$ ).

\section{Discussion}

Our study confirmed that the Q-Sticks test might be considered as a screening tool that enables preliminary diagnosis of anosmia. The data indicated a test sensitivity and specificity of 91.8 and $92 \%$, respectively, with a score of 2 taken as a cutoff for anosmia. Conversely, normal olfactory function can be declared with high probability with a score of 3 in the Q-Sticks test. Our study showed that the test can be used on subjects aged 12 years and older, regardless of their sex. The Q-Sticks test is expected to be useful and convenient as a screening tool for olfactory impairments (Fig. 1). It should be noted, however, that with the cutoff score of 2 , a certain percentage of anosmic subjects will not be recognized (in the present case about $8 \%$ of functionally anosmic patients) and that it yields a relatively high percentage of false alarms, as some normosmic subjects in our study obtained scores $<3$. On the other hand, although the test is particularly good to identify patients with abnormal olfactory function if the score is 0 , it needs to be kept in mind that the inverse case is not true: almost one-third of patients with a score of 3 have abnormal olfactory function. Thus, the Q-Sticks test cannot replace solid olfactory tests based on more items or other testing methodology, e.g., the 16-item odor identification test or the extended version of the Sniffin' Sticks, which allows for the follow-up of patients with olfactory loss. The major advantages of the Q-Sticks test relate to its simplicity, speed of application, portability, and that it can be reused. As mentioned above, it is a screening tool which is very user-friendly in daily routine, and it might be used as a preliminary diagnostic tool, especially for anosmia (Fig. 1).

The current multicenter study does not change the proposed application of this test and the consequences drawn from the results of the Q-Sticks test as proposed previously [17] (Fig. 1). Specifically, if the patient scores 3, no further olfactory assessment appears to be 
Sorokowska et al.: Olfactory Screening with the Q-Sticks Test

necessary. If the patient scores 1 or 2 , detailed olfactory testing should be performed depending on the patient's history, the patient's complaints, and the physician's judgement. A score of 0 should prompt more detailed olfactory investigations. Again, when performing this routine it should always be kept in mind that a small portion of anosmic patients may just pass this screening.

Olfactory problems are often among the first signs of neurodegenerative diseases [2], and general practitioners might wish to monitor the olfactory abilities of their patients during regular checkups. In fact, changes in olfactory function may provide important clinical information [18]. The easiest way to monitor such changes seems to be self-assessment by the patient, and in fact, clinicians often rely on short, unstructured interviews to assess the extent of a patient's problem. However, self-assessments are only moderately related to actual olfactory sensitivity [19]. Negligence of olfactory dysfunction seems to be also very common in the general population [20]. This seems to be particularly important in subjects who are not aware of their decreased olfactory function, as is often the case in patients with, e.g., idiopathic Parkinson's disease [2]. In such cases, short tests such as the Q-Sticks test might be good alternative screening tools.

\section{Conclusion}

In summary, there are numerous difficulties associated with the classical, longer tests in everyday practice (time-consuming, expensive, not reusable, and difficult to perform with children and elderly adults). However, the low reliability of self-reported olfactory function hints the importance of more objective tests of olfactory function. We reconfirmed that the Q-Sticks test is a useful screening tool allowing to identify patients with olfactory disorders with high probability. The test was relatively sensitive to anosmia and differences between normosmic and hyposmic/anosmic patients. Therefore, when time is limited and the olfactory test is meant to be a simple, rough estimate of olfactory sensitivity, the Q-Sticks test seems to be a very good solution.

\section{Statement of Ethics}

Investigations were performed according to the Guidelines for Biomedical Studies Involving Human Subjects ("Helsinki Declaration"). The protocol was approved by the Ethics Committee of the Medical Faculty of the TU Dresden. All subjects provided written informed consent prior to their inclusion in the study.

\section{Disclosure Statement}

The authors declare no potential conflicts of interest with respect to the research, authorship, and/or publication of this article.

\section{Funding Sources}

The research was supported by funds of the Polish Ministry of Science and Higher Education (A. Sorokowska: scholarship for the years 2013-2016 and Iuventus Plus grant \#IP2014 043773 for the years 20152017; A. Oleszkiewicz: scholarship \#626/STYP/12/2017). 
Sorokowska et al.: Olfactory Screening with the Q-Sticks Test

\section{References}

1 Landis BN, Konnerth CG, Hummel T. A study on the frequency of olfactory dysfunction. Laryngoscope. 2004 Oct; 114(10):1764-9.

2 Haehner A, Hummel T, Hummel C, Sommer U, Junghanns S, Reichmann H. Olfactory loss may be a first sign of idiopathic Parkinson's disease. Mov Disord. 2007 Apr;22(6):839-42.

3 Croy I, Symmank A, Schellong J, Hummel C, Gerber J, Joraschky P, et al. Olfaction as a marker for depression in humans. J Affect Disord. 2014 May;160:80-6.

4 Jiang T, Soussignan R, Schaal B, Royet JP. Reward for food odors: an fMRI study of liking and wanting as a function of metabolic state and BMI. Soc Cogn Affect Neurosci. 2015 Apr;10(4):561-8.

5 Bartolo M, Serrao M, Gamgebeli Z, Alpaidze M, Perrotta A, Padua L, et al. Modulation of the human nociceptive flexion reflex by pleasant and unpleasant odors. Pain. 2013 Oct;154(10):2054-9.

6 Chen D, Haviland-Jones J. Rapid mood change and human odors. Physiol Behav. 1999 Dec;68(1-2):241-50.

7 Doty RL. Olfactory dysfunction and its measurement in the clinic and workplace. Int Arch Occup Environ Health. 2006 Apr;79(4):268-82.

8 Hummel T, Sekinger B, Wolf SR, Pauli E, Kobal G. "Sniffin' sticks": olfactory performance assessed by the combined testing of odor identification, odor discrimination and olfactory threshold. Chem Senses. 1997 Feb; 22(1):39-52.

9 Hummel T, Kobal G, Gudziol H, Mackay-Sim A. Normative data for the "Sniffin' Sticks" including tests of odor identification, odor discrimination, and olfactory thresholds: an upgrade based on a group of more than 3,000 subjects. Eur Arch Otorhinolaryngol. 2007 Mar;264(3):237-43.

10 Sorokowska A, Albrecht E, Haehner A, Hummel T. Extended version of the “Sniffin' Sticks” identification test: test-retest reliability and validity. J Neurosci Methods. 2015 Mar;243:111-4.

11 Landis BN, Hummel T. New evidence for high occurrence of olfactory dysfunctions within the population. Am J Med. 2006 Jan;119(1):91-2.

12 Doty RL, Shaman P, Dann M. Development of the University of Pennsylvania Smell Identification Test: a standardized microencapsulated test of olfactory function. Physiol Behav. 1984 Mar;32(3):489-502.

13 Hedner M, Larsson M, Arnold N, Zucco GM, Hummel T. Cognitive factors in odor detection, odor discrimination, and odor identification tasks. J Clin Exp Neuropsychol. 2010 Dec;32(10):1062-7.

14 Hummel T, Konnerth CG, Rosenheim K, Kobal G. Screening of olfactory function with a four-minute odor identification test: reliability, normative data, and investigations in patients with olfactory loss. Ann Otol Rhinol Laryngol. 2001 Oct;110(10):976-81.

15 Kobal G, Hummel T, Sekinger B, Barz S, Roscher S, Wolf S. "Sniffin' sticks”: screening of olfactory performance. Rhinology. 1996 Dec;34(4):222-6.

16 Mueller C, Renner B. A new procedure for the short screening of olfactory function using five items from the “Sniffin' Sticks" identification test kit. Am J Rhinol. 2006 Jan-Feb;20(1):113-6.

17 Hummel T, Pfetzing U, Lötsch J. A short olfactory test based on the identification of three odors. J Neurol. 2010 Aug;257(8):1316-21.

18 Gudziol V, Lötsch J, Hähner A, Zahnert T, Hummel T. Clinical significance of results from olfactory testing. Laryngoscope. 2006 Oct;116(10):1858-63.

19 Sorokowska A, Schriever VA, Gudziol V, Hummel C, Hähner A, Iannilli E, et al. Changes of olfactory abilities in relation to age: odor identification in more than 1400 people aged 4 to 80 years. Eur Arch Otorhinolaryngol. 2015 Aug;272(8):1937-44.

20 Wehling E, Lundervold AJ, Espeset T, Reinvang I, Bramerson A, Nordin S. Even cognitively well-functioning adults are unaware of their olfactory dysfunction: implications for ENT clinicians and researchers. Rhinology. 2015 Mar;53(1):89-94. 